\title{
The Effect of Roux-en-Y vs. Omega-Loop Gastric Bypass on Liver, Metabolic Parameters, and Weight Loss
}

\author{
Renate Kruschitz $^{1,5}$ - Maria Luger ${ }^{1,2} \cdot$ Christian Kienbacher $^{3} \cdot$ Michael Trauner $^{3}$. \\ Carmen Klammer ${ }^{1}$ - Karin Schindler ${ }^{1}$. Felix B. Langer ${ }^{4}$ - Gerhard Prager ${ }^{4}$. \\ Michael Krebs $^{1} \cdot$ Bernhard Ludvik $^{1,5}$
}

Published online: 22 March 2016

(C) The Author(s) 2016. This article is published with open access at Springerlink.com

\begin{abstract}
Background Omega-loop gastric bypass (OLGB) results in weight loss (WL) but data on its impact on liver and glucose metabolism compared to Roux-en-Y gastric bypass (RYGB) is lacking. Therefore, the aim of this study was to compare the development of hepatic and metabolic markers as well as WL between the above-mentioned surgical groups during the first postoperative year.

Methods We retrospectively evaluated the respective parameters in non-diabetic morbidly obese patients who underwent either RYGB $(n=25)$ or OLGB $(n=25)$.

Results Compared to RYGB, OLGB showed a greater WL percentage. Liver transaminases dropped in RYGB, while rose in OLGB. No correlation between aspartate
\end{abstract}

Renate Kruschitz and Maria Luger contributed equally to this work.

Renate Kruschitz

renate@kruschitz.de

Maria Luger

maria.luger@meduniwien.ac.at

Christian Kienbacher

christian.a.kienbacher@meduniwien.ac.at

Michael Trauner

michael.trauner@meduniwien.ac.at

Carmen Klammer

Carmen.Klammer@gmx.at

Karin Schindler

karin.schindler@meduniwien.ac.at

Felix B. Langer

felix.langer@meduniwien.ac.at

Gerhard Prager

gerhard.prager@meduniwien.ac.at transaminase, alanine transaminase, and WL could be detected. Gamma-glutamyltransferase decreased significantly in RYGB over the first 3 months, while it increased in OLGB. We found higher levels of triglycerides, insulin, homeostasis model assessment of insulin resistance (HOMA2-IR), and liver fat percentage in RYGB at baseline, despite matching the groups for age, sex, and BMI. Those differences disappeared, except for triglycerides, within 1 year. All metabolic parameters correlated with WL.

Conclusion OLGB results in greater WL but transiently deteriorated several liver parameters in the first postoperative year. This was not associated with WL. The impact of these results on hepatic outcomes such as non-alcoholic steatohepatitis and fibrosis progression

Michael Krebs

michael.krebs@meduniwien.ac.at

Bernhard Ludvik

bernhard.ludvik@wienkav.at

Division of Endocrinology and Metabolism, Department of Internal Medicine III, Medical University of Vienna, Vienna, Austria

2 Special Institute for Preventive Cardiology And Nutrition - SIPCAN save your life, Salzburg, Austria

3 Division of Gastroenterology and Hepatology, Department of Internal Medicine III, Medical University of Vienna, Vienna, Austria

4 Division of General Surgery, Department of Surgery, Medical University of Vienna, Vienna, Austria

5 Department of Internal Medicine I with Diabetology, Endocrinology and Nephrology Rudolfstiftung Hospital, Karl Landsteiner Institute for Obesity and Metabolic Diseases, Vienna, Austria 
requires further studies. In both groups, improved insulin resistance and sensitivity were correlated with higher WL and lower liver fat percentage, respectively.

Keywords Gastric bypass $\cdot$ Morbid obesity $\cdot$ Liver parameters $\cdot$ Metabolic parameters $\cdot$ Weight loss

\section{Introduction/Purpose}

Bariatric surgery is the most successful treatment of morbid obesity [1] since it is associated with effective long-term weight loss (WL) and decreases overall mortality [2]. At present, three categories of bariatric procedures are in use: (a) purely gastric restriction (e.g., gastric banding, sleeve gastrectomy); (b) gastric restriction with a mild malabsorptive effect, represented by Roux-en-Y gastric bypass and omega-loop gastric bypass; and (c) gastric restriction with an extensive malabsorptive effect such as the biliopancreatic diversion [3]. The extent of WL is in part related to the complexity of the bariatric procedure [4]. A small number of studies have compared the results of Roux-en-Y gastric bypass, which is still the gold standard for many bariatric teams [5], and omega-loop gastric bypass. Regarding previous data, it became evident that omega-loop gastric bypass is a bariatric procedure that demonstrates initial promising results in terms of WL, feasibility, and safety [6, 7]. However, little is known about the short- and long-term impact of this procedure, especially in terms of its impact on liver function and glucose metabolism.

Several studies investigating the effect of bariatric surgery on liver enzymes and non-alcoholic fatty liver disease (NAFLD) have shown an improvement of serum transaminases and hepatic histologic features after surgery [8-11]. From previous data, however, it became evident that rapid $\mathrm{WL}$, as seen with bariatric surgery, can also adversely affect the liver [11]. Moreover, several case reports of patients with early hepatic failure after bariatric surgery are described in the literature [12-14].

The aim of this evaluation was to provide data on the development of hepatic and metabolic markers as well as on WL in morbidly obese patients undergoing either Roux-en-Y gastric bypass or omega-loop gastric bypass during the first 12 postoperative months to facilitate the design of studies in larger populations.

\section{Materials and Methods}

\section{Preoperative Evaluation}

For this investigation, we accomplished an analysis within the cohort study considering all consecutive bariatric patients in the outpatient clinic of the Division of Endocrinology and Metabolism in the General Hospital Vienna. The procedures used in this study were in accordance with the Declaration of Helsinki [15] and were approved and registered (no. 988/ 2011) by the Ethics Committee of the Medical University of Vienna. All participants provided their written informed consent prior to the study. A multidisciplinary team performed a combined workup to ensure that potential surgical candidates met the criteria for bariatric surgery [16]. The exclusion criteria were as follows: diabetes, earlier bariatric surgery, active malignancy during the past 5 years, myocardial infarction during the past 6 months, eating disorders, psychiatric problems contraindicating bariatric surgery, regular use of cortisone, alcohol ( $>20 \mathrm{~g} /$ day men, $>10 \mathrm{~g} /$ day women) or drug abuse, and other severe illnesses. Daily alcohol consumption was evaluated during the preoperative dietary counseling on basis of the self-reported nutritional protocols.

\section{Patients}

From February 2011 to February 2013, a total of 86 patients underwent omega-loop gastric bypass [17]. Due to results on preliminary data that showed significant differences between diabetic and non-diabetic subjects [18], diabetic patients $(n=22)$ were not included in this analysis. In order to adequately compare the available data on omega-loop gastric bypass to the Roux-en-Y gastric bypass procedure, we matched patients by age, gender, and initial body mass index (BMI). Moreover, 36 patients with missing preoperative liver data and 3 with no adequate Roux-en-Y gastric bypass matches were excluded from the current analysis. Non-diabetic subjects who underwent either Roux-en-Y gastric bypass $(n=25$, female $=92 \%, 44.6 \pm 10.3 \mathrm{a}, 125 \pm 18 \mathrm{~kg}$, BMI $45.6 \pm 4.1)$ or omega-loop gastric bypass $(n=25$, male $=88 \%, 43.8 \pm 13.1 \mathrm{a}, 128 \pm 24 \mathrm{~kg}$, BMI $45.3 \pm 5.3)$ were analyzed. Baseline data are presented in Table 1 and Figs. 1, 2, 3, and 4.

\section{Surgical Technique}

All procedures were performed by the same surgical team using a laparoscopic approach. Roux-en-Y gastric bypass consists of a longitudinal 30-ml gastric pouch which is anastomosed end-to-side with the jejunal limb and a latero-lateral jejuno-jejunal anastomosis resulting in a biliopancreatic limb of approximately $80 \mathrm{~cm}$ and an alimentary limb of approximately $150 \mathrm{~cm}$ [5]. Omega-loop gastric bypass is a simplified procedure that consists of a unique gastrojejunal anastomosis between a 30-40-ml sleeve gastric pouch and a jejunal omega loop of $200 \mathrm{~cm} \mathrm{[5,19].}$ 


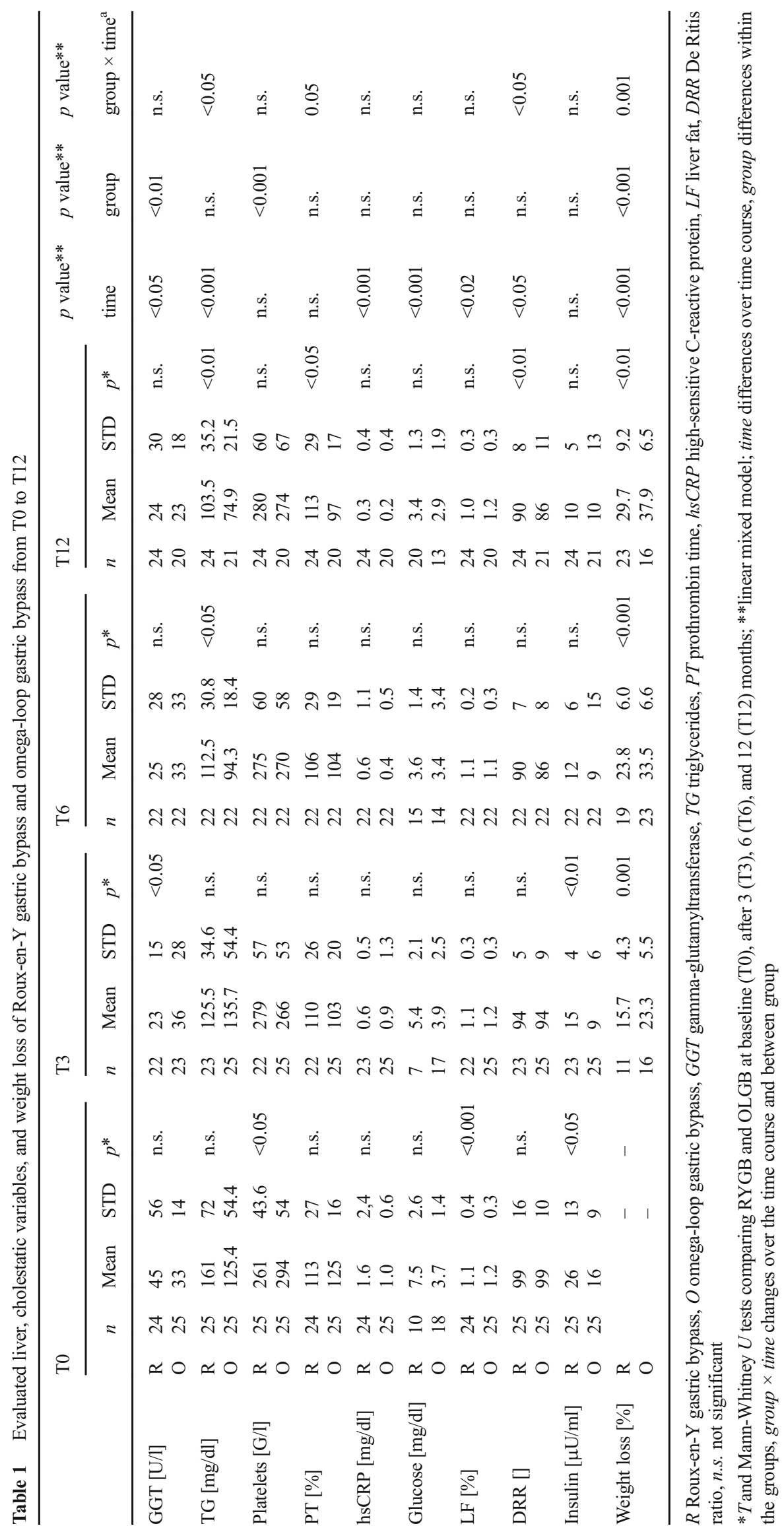




\section{Follow-Up}

All patients were followed up before surgery ( $\mathrm{T} 0$ ) (on average 2 months), 3 (T3), 6 (T6), and 12 (T12) months postoperatively. Medical treatment was adjusted according to the current needs of the patient. Postoperatively, supplements were prescribed with respect to the available guidelines at that time [3].

\section{Variables}

Following blood parameters were evaluated: total protein, albumin, total bilirubin, alkaline phosphatase (AP), aspartate transaminase (AST), alanine transaminase (ALT), gammaglutamyltransferase (GGT), triglycerides (TG), prothrombin time, platelets, glucose, insulin, and high-sensitive C-reactive protein (hsCRP) as marker for tissue damage, infection, inflammation, and malignant neoplasia [20]. The NAFLD liver fat score [21], a test to accurately predict non-alcoholic fatty liver disease (NAFLD), liver fat percentage (\%) [21], based on fasting glucose, fasting insulin, De Ritis ratio, the presence of metabolic syndrome and type 2 diabetes, and the NAFLD fibrosis score [22], a scoring system consisting of BMI, age, glycemic status, platelet count, albumin level, and De Ritis ratio [22] to separate NAFLD patients with and without advanced fibrosis were calculated. Furthermore, De Ritis ratio, as a marker for severe liver damage [23], as well as homeostasis model assessment of insulin resistance (HOMA2-IR) and quantitative insulin sensitivity check index (QUICKI) [24], as marker for insulin sensitivity, were computed. The presence of metabolic syndrome was defined according to criteria of the International Diabetes Federation [25]. Abnormal liver parameters were defined as an elevation of ALT, and/or AST, and/or GGT levels above the upper limit of normal (ULN) [26]. In order to discuss the clinical relevance of any findings, there was a separate analysis with values $>2 \times$ ULN and the proportion of results out of the normal range. Age, height, weight, and BMI were determined. Due to the different WL reporting methods in previous studies, we decided to calculate all of the recommended ones [27] to allow easier comparison of the data.

\section{Statistical Analysis}

Statistical calculations were performed by SPSS 20.0 (SPSS Inc, Chicago, IL). Data are shown as means and standard deviations (SD). The hypothesis of variables being normally distributed was tested by Kolmogorov-Smirnov test. Differences in the distributions of variables between Roux-en-Y gastric bypass and omega-loop gastric bypass were tested by a Student's $t$ test, respectively, by Mann-Whitney $U$ test for two independent samples. We used repeated-measures ANOVA, using random error (linear mixed model) to assess the effect of time and the interaction for changes in laboratory parameters between the groups, by using different covariance structure models as appropriate and adjusted for baseline values, age, and sex. Moreover, a post hoc analysis with Bonferroni correction was used. Binary logistic regression was used to estimate the odds for type of surgery. Linear regression was used to identify independent variables (e.g., age, gender, preoperative BMI) associated with changes in the evaluated parameters. Spearman's and Pearson's correlation coefficients were used to determine associations between changes in relevant parameters. The chi-square test was applied to control whether there are significant differences between the expected and the observed frequencies in categorical liver, cholestatic, and metabolic parameters. Means were compared unadjusted without imputation of missing data. Statistical significance for all analyses was assumed as $p \leq 0.05$.

\section{Results}

\section{Liver Function}

For AST, a significant group and time difference could be found. ALT dropped in Roux-en-Y gastric bypass, while rising in omega-loop gastric bypass with a significant group and time difference. Moreover, a positive correlation could be found between ALT, surgical method $(r=0.406, p=0.006)$ and De Ritis ratio $>1(r=0.451, p=0.002)$ at T12. Notably, no correlation between WL, ALT, and AST was observed. These parameters remained in the normal range in both groups (Fig. 1).

Initially, Omega-loop gastric bypass group showed a significant higher count in platelets, this difference disappeared at T3. Nevertheless, a difference between the groups (Table 1), as well as a negative correlation with WL $(r=-0.346$, $p=0.002$ ) could be detected.

A significant group and time interaction could be found for prothrombin time (Table 1) as well as a significant increasing proportion of low prothrombin time in omega-loop gastric bypass at T3 (Roux-en-Y gastric bypass vs. omega-loop gastric bypass; T0: 8 vs. $0 \%$, n.s.; T3: 5 vs. $8 \%, p<0.05$ ). A negative correlation between prothrombin time and WL was found in omega-loop gastric bypass $(r=-0.543, p<0.001)$.

No differences appeared in the course of albumin, while protein diminished significantly over time and group (Fig. 2). A negative correlation between protein and WL in both groups $(r=-0.428, p<0.001)$, as well as for albumin and hsCRP $(r=-0.611, p<0.01)$ in omega-loop gastric bypass at T6 was found.

\section{Cholestatic Parameters}

In Roux-en-Y gastric bypass, GGT significantly decreased by nearly half of the initial value, while it remained stable in omega-loop gastric bypass and a significant group and time 
Fig. 1 Pre- and postoperative mean protein and albumin levels in patients with Roux-en-Y gastric bypass (marked by a full line) and omega-loop gastric bypass (marked by a dotted line). ${ }^{a} T$ test comparing Roux-en-Y gastric bypass and omega-loop gastric bypass at baseline (T0), 3 (T3), 6 (T6), and 12 (T12) months. Linear mixed model (LMM), adjusted for baseline values, sex, and age for changes over ${ }^{b}$ time course, ${ }^{c}$ between groups, and $d_{\text {changes over time course and }}$ between groups. $* p<0.05$; $* * p<0.01 ; * * * p<0.001$; n.s. not significant

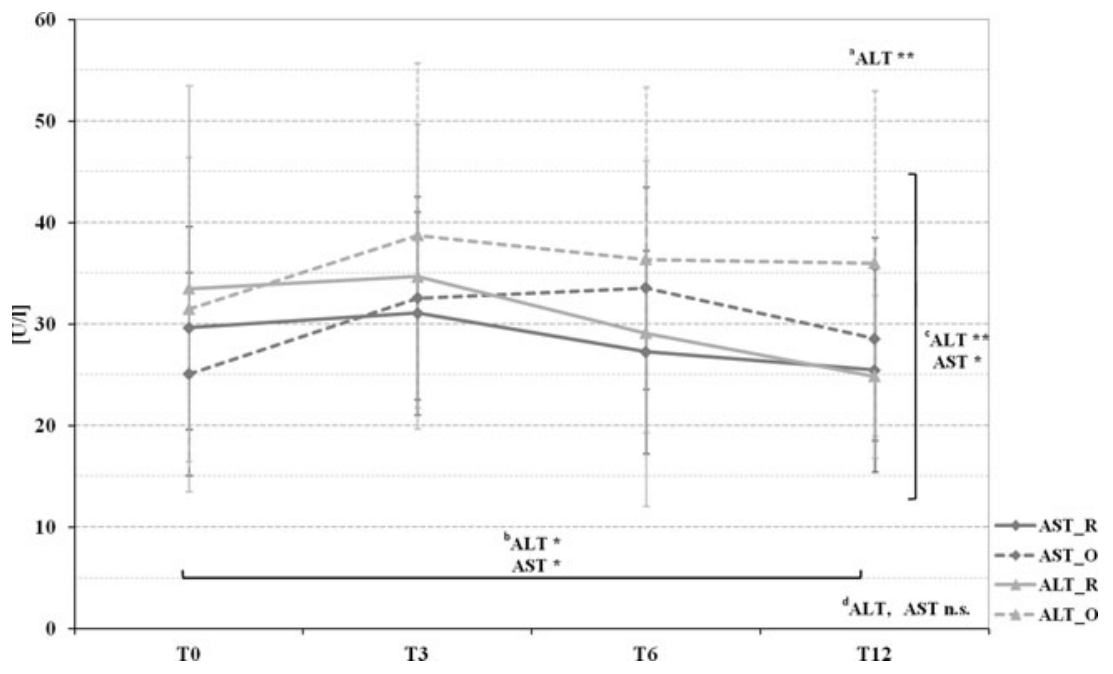

difference could be found (Table 1). Younger patients $(<50$ years $)$ had a greater chance of lower GGT quartiles over time (odds ratio (OR) $0.52 ; 95 \%$ confidence interval $(\mathrm{CI})=0.36-0.75 ; p<0.001$; adjusted for initial BMI). A negative correlation between GGT and WL could be seen in Roux-en-Y gastric bypass $(r=-0.571, p<0.001)$.

In the whole cohort, a maximum rise in bilirubin and AP was found at T3. The values of both remained in the highnormal range until T12 (Fig. 3).

\section{Calculated Liver Scores}

NAFLD liver fat score showed a significant higher proportion of NAFLD in Roux-en-Y gastric bypass until T6 (T0: Rouxen-Y gastric bypass $75 \%$ vs. omega-loop gastric bypass $6 \%$, $p<0.001$; T3: 57 vs. $8 \%, p<0.01$; T6: 13 vs. $5 \%$, n.s.; T12: 0 vs. $13 \%$, n.s.).

Liver fat percentage showed a similar development with a significant difference over time (Table 1). Over the whole observation period, a strong correlation could be found between liver fat percentage and HOMA2-IR $(r=0.616$, $p<0.001)$; QUICKI $(r=-0.623 p<0.001)$; AST $(r=0.506$, $p<0.001)$; ALT $(r=0.429, p<0.001)$; and TG (T0: $r=0.519, p<0.001)$ in both groups.

Omega-loop gastric bypass showed a significant higher $D e$ Ritis ratio at T12 (Table 1) and a significant group and time interaction could be detected. Subsequently, at T12, $54 \%$ in Roux-en-Y gastric bypass vs. $90 \%$ in the omega-loop gastric bypass $(p<0.01)$ had a De Ritis ratio $\geq 1$. Over the observation period, no differences were found in the distribution of individuals with, without, and undefined status of liver fibrosis calculated by NAFLD fibrosis score.

\section{Metabolic Markers}

Glucose significantly decreased in both groups until T12. Insulin was significantly higher in Roux-en-Y gastric bypass at $\mathrm{T} 0$, although no group and time interaction could be found.
Fig. 2 Pre- and postoperative mean AST and ALT levels in patients with Roux-en-Y gastric bypass (marked by a full line) and omega-loop gastric bypass (marked by a dotted line). ${ }^{a} T$ test comparing Roux-en-Y gastric bypass and omega-loop gastric bypass at baseline (T0), 3 (T3), 6 (T6), and 12 (T12) months. Linear mixed model (LMM), adjusted for baseline values, sex, and age for changes over ${ }^{b}$ time course, ${ }^{c}$ between groups, and ${ }^{d}$ changes over time course and between groups. $* p<0.05$; ${ }^{* *} p<0.01 ; * * * p<0.001$; n.s. not significant

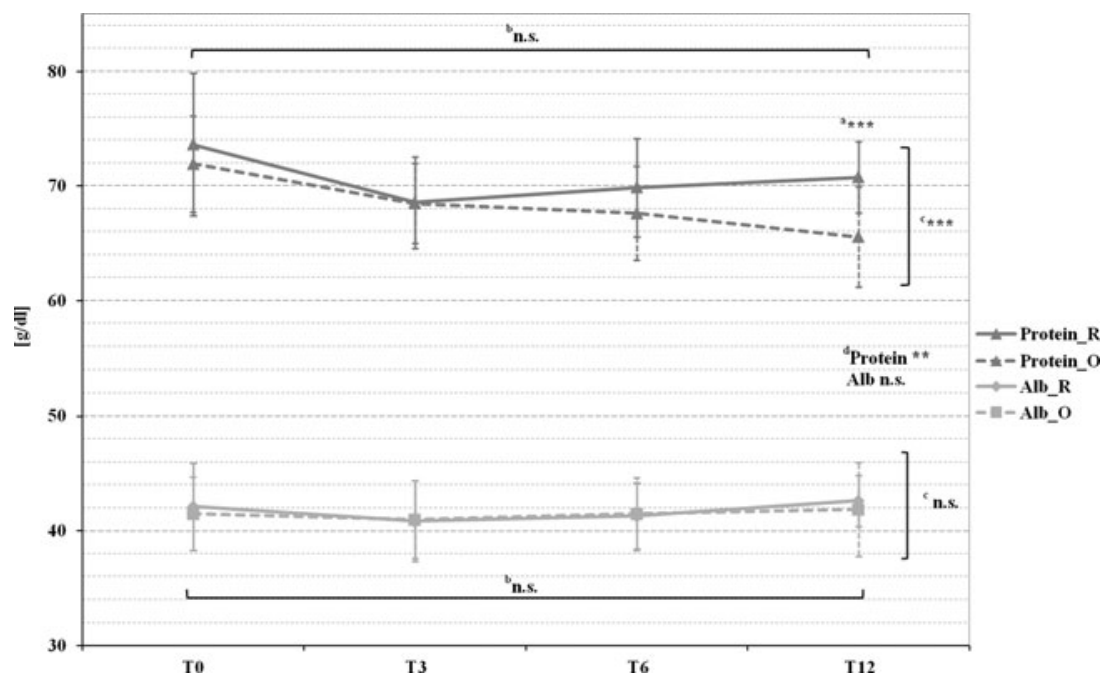


Fig. 3 Pre- and postoperative mean bilirubin and alkaline phosphatase levels in patients with Roux-en-Y gastric bypass (marked by a full line) and omega-loop gastric bypass (marked by a dotted line). ${ }^{a} T$ Test comparing Roux-en-Y gastric bypass and omega-loop gastric bypass at baseline (T0), 3 (T3), 6 (T6), and 12 (T12) months. Linear mixed model (LMM), adjusted for baseline values, sex, and age for changes over ${ }^{b}$ time course, ${ }^{c}$ between groups, and ${ }^{d}$ changes over time course and between groups. ${ }^{*} p<0.05$; ${ }^{* *} p<0.01$; *** $p<0.001$; n.s. not significant

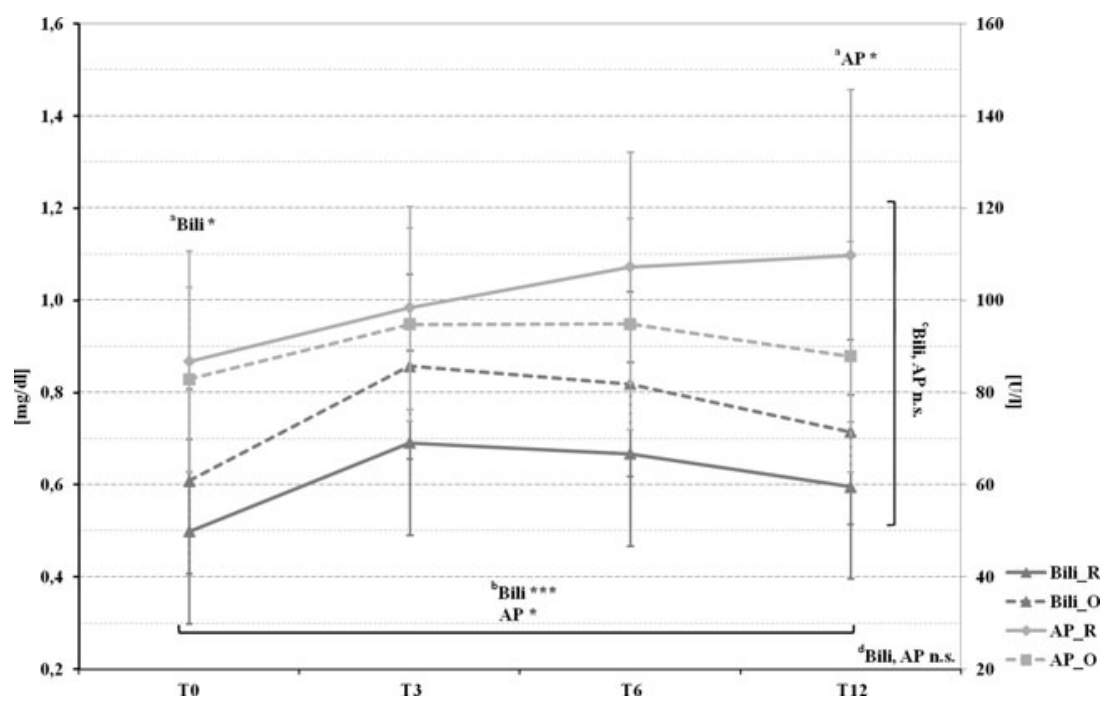

We observed a significant group difference for HOMA2-IR from T0 to T6 and a significant group and time effect (Fig. 4), whereby values were higher in Roux-en-Y gastric bypass. QUICKI was significantly higher in omega-loop gastric bypass patients at T3, T6, and T12; however, no group and time interaction could be detected (Fig. 4).

TG showed a significant reduction over time in both groups. Worth mentioning is a significant initial increase of TG in omega-loop gastric bypass, which finally ended up in a greater reduction. For all above-mentioned metabolic markers, a negative, respectively, for QUICKI positive, correlation with WL in both groups could be seen (glucose: $r=-0.451, p<0.001$; insulin: $r=-0.379, p<0.001$; HOMA2-IR: $r=-0.520, p<0.001$; QUICKI: $r=0.540$, $p<0.001$; TG: $r=-0.418, p<0.001$ ).

hsCRP showed a reduction over time with lowest levels in both groups at T12. Notable correlations were found with WL $(r=-0.293, p<0.001)$ over time in both groups, respectively. AST $(r=0.527, p<0.001)$; AST $>2 \times \operatorname{ULN}(r=0.501$, $p=0.001)$; ALT $(r=0.358, p=0.02)$; ALT $>2 \times \mathrm{ULN}$ $(r=0.488, p<0.01)$; and glucose $(r=0.474, p<0.01)$, in both groups at $\mathrm{T} 0$.

\section{Weight Loss}

$\mathrm{WL}$ (mean $\pm \mathrm{SD}$ ) was $30 \pm 9$ vs. $38 \pm 7 \%$ (\%BMI loss), which equals a percentage excess WL (\%EWL) of $94 \pm 36$ vs. 127 $\pm 31 \%$ or percentage excess BMI loss (\%EBMIL) of $67 \pm 22$ vs. $88 \pm 16 \%$ in Roux-en-Y gastric bypass vs. omega-loop gastric bypass after 12 months. Using the binary logistic regression, an increased chance for greater WL in omega-loop gastric bypass was shown $(\mathrm{OR}=1.19 ; 95 \% \mathrm{CI}=1.11-1.27$; $p<0.001)$.
Fig. 4 Pre- and postoperative mean HOMA2-IR and QUICKI levels in patients with Roux-en-Y gastric bypass (marked by a full line) and omega-loop gastric bypass (marked by a dotted line). ${ }^{a} T$ test comparing Roux-en-Y gastric bypass and omega-loop gastric bypass at baseline (T0), 3 (T3), 6 (T6), and 12 (T12) months. Linear mixed model (LMM), adjusted for baseline values, sex, and age for changes over ${ }^{b}$ time course, ${ }^{c}$ between groups, and ${ }^{d-}$ changes over time course and between groups. $* p<0.05$; $* * p<0.01 ; * * * p<0.001 ;$ n.s. not significant

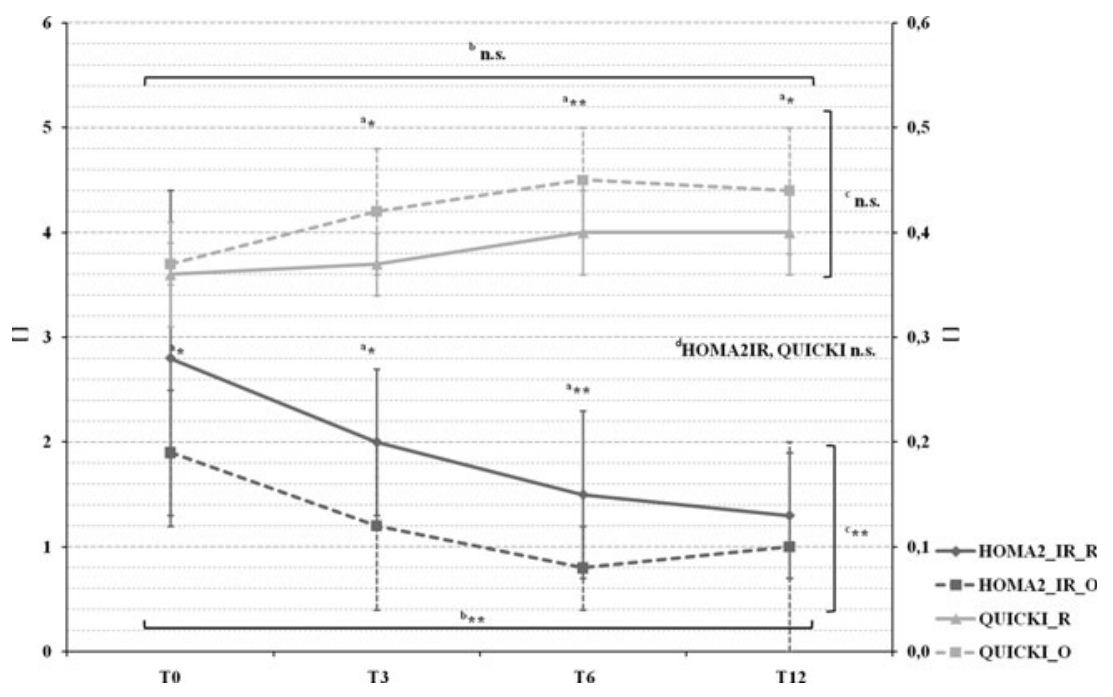




\section{Discussion}

The superiority of omega-loop gastric bypass over Roux-en-Y gastric bypass in terms of WL was demonstrated in previous studies $[5,6]$, as well as in our study. We did not observe any influence of gender, age, or preoperative BMI on WL regardless of the procedure. Studies reported an EWL percentage range from 59 to $63 \%$ in Roux-en-Y gastric bypass [5-7, $19,28-30]$ and 65 to $89 \%$ in omega-loop gastric bypass [5, $6,30]$. Compared to this data, we observed a larger WL in both groups. The difference might be explained by differences in the length of the bypassed small bowel with a differing malabsorptive effect. In addition to the superior WL in omega-loop gastric bypass, we might assume additional beneficial effects, as elevated transaminases, cholestatic and metabolic parameters improve driven by WL $[11,31,32]$.

WL is currently an effective treatment of NAFLD and non-alcoholic steatohepatitis (NASH) in patients with obesity [11, 32, 33]. Several studies investigating the effect of bariatric surgery on liver enzymes and NAFLD have shown an improvement of serum transaminases and hepatic histologic features after surgery [8-11]. However, so far, no current data is available for omega-loop gastric bypass and liver status. Certainly from previous literature, it became evident that rapid WL after bariatric surgery can also adversely affect the liver [11]. This could be attributed to the already pre-existing degree of fibrosis which subsequently worsened by rapid WL due to the bariatric procedure and/or due to a lack of adequate supplementation of macro- and micronutrients [11, 34].

In our study, the increase of liver transaminases (ALT, AST) was greater in omega-loop gastric bypass than in Roux-en-Y gastric bypass with a maximum rise after 3 months. These findings are comparable with two studies of Wolf AM et al. [9, 35]. The values of AST and ALT successively decreased and finally fell below those of $\mathrm{T} 0$ in Roux-en-Y gastric bypass, whereas both parameters remained above the preoperative values in omega-loop gastric bypass. The results remained within the normal range in both groups and during the whole observation period. Nevertheless, even slightly increased but still normal aminotransferase concentrations are related to an increased risk of death from liver disease [36]. Significant liver disease (i.e., bridging fibrosis and cirrhosis) may be accompanied by mild transaminase elevations [37], as seen in our data. GGT, as well as AST, are associated with higher risk of cardiovascular disease (CVD), particularly in young individuals $[38,39]$. GGT is known as a marker for sub-clinical inflammation, oxidative stress, visceral, respectively, intrahepatic fat and therefore for insulin resistance [38]. Our data shows a rapid reduction of GGT in Roux-en$\mathrm{Y}$ gastric bypass and a temporally delayed one in omega-loop gastric bypass, especially in younger patients, who might benefit most from a reduction [39]. Bilirubin and AP were both increasing after surgery and remained above the baseline level after 12 months. Moreover, a dependence of bilirubin and AP with WL could only be seen in Roux-en-Y gastric bypass. These findings are contrary to previous data [40]. Furthermore, we found a clear positive association between $\mathrm{WL}$ and the improvement of parameters for insulin resistance, insulin sensitivity and liver fat percentage in both groups. Interestingly, in comparison to other studies [8,32], we could not find a correlation between AST, ALT, and WL.

Noteworthy, the proportion of patients with higher De Ritis ratio was significantly greater in omega-loop gastric bypass after 12 months. Furthermore, omega-loop gastric bypass presented a significantly higher level of ALT, lower level of protein with a negative correlation with WL, prothrombin time, and platelets, which are also indicators for liver function [41], at T12. The transient elevation of liver parameters in patients after bariatric surgery $[11,34,35]$ and during a low-calorie diet without bariatric surgery [31] were previously described. Different hypotheses explaining these changes in hepatic and metabolic parameters exist, whereas it is most likely that the triggers are multifactorial. Mild and transient changes in liver histology, which themselves could be induced by the modifications in liver physiology such as the increased ketone production or active mobilization of lipids from adipose tissue and hepatocytes during calorie restriction resulting in potentially toxic intermediate metabolites such as free fatty acids and ceramides, could be a key mechanism [31, 42]. Furthermore, rapid mobilization of intra- and extrahepatic fat stores are probably associated with deficient protein intake during WL and may contribute to aggravation of preexisting liver disease [34]. Moreover, the trauma of surgery associated with an increase in production of stress hormones, oxidative stress, and inflammatory responses is associated with reduced hepatic function [9]. Importantly, we could not detect a correlation between liver transaminases and WL.

We found higher levels of several metabolic parameters (insulin, HOMA2-IR, liver fat percentage) at T0 in Roux-en$\mathrm{Y}$ gastric bypass despite of matching our groups for age, sex, and BMI. Those differences disappeared at T12, and all of them were positively correlated with WL. Consequently, both surgical groups showed a positive metabolic outcome due to WL.

A limitation of the current report is the small sample size, due to the restricted availability of non-diabetic omega-loop gastric bypass data. Nevertheless, this sample is well characterized and the laboratory data is almost complete, which strengthens our study. The data represents a novelty, because up to now, to our current knowledge, no study has assessed the differences in liver, cholestatic, and metabolic parameters in Roux-en-Y gastric bypass vs. omega-loop gastric bypass. Another limitation is the lack of liver biopsies, which might give us better insight into the liver situation preoperatively and 
during the postoperative period. Therefore, we cannot exclude the presence of relevant liver injury at baseline, which possibly could explain the results in omega-loop gastric bypass at T12.

In conclusion, omega-loop gastric bypass results in greater WL and deterioration of liver parameters in the first year after surgery with no association to WL. It remains unclear what induces the bimodal alteration in liver transaminases and cholestatic parameters during the early postoperative phase and also after 12 months. Given the potential for worsening fibrosis following bariatric surgery, patients should be monitored closely and continue to undergo through hepatological workup, including non-invasive testing for fibrosis or, even liver biopsy. More research in this field, including histopathological data, is needed to define clinical relevance of these findings on hepatic outcome in terms of deterioration of non-alcoholic steatohepatitis and fibrosis and to characterize patients at risk. In both groups, improved outcome in terms of insulin resistance and sensitivity could be found in correlation to higher WL and lower liver fat percentage, respectively.

Authors' contributions The authors' responsibilities were as follows: FL, BL, KS, and GP designed the research. ML, RK, and CK collected the data and performed statistical analyses. RK and ML drafted the manuscript with appreciable input from BL, CHK, MT, MK, FL, KS, and GP. RK and ML had prime responsibility for the final manuscript content. All authors read and approved the final manuscript.

\section{Compliance with Ethical Standards}

Conflict of Interest Statement RK, ML, CHK, CK, FL, BL, KS, and MK declare that they have no conflict of interests in the preparation of this manuscript. MT has received advisory board and lecture fees from Albireo, BMS, Falk, Gilead, Intercept, Jannsen, MSD, and Roche, and he has received unrestricted research grants from Albireo, Falk, Intercept, and MSD. GP reports grants from Educational Grant Medtronic outside the submitted work.

Open Access This article is distributed under the terms of the Creative Commons Attribution 4.0 International License (http:// creativecommons.org/licenses/by/4.0/), which permits unrestricted use, distribution, and reproduction in any medium, provided you give appropriate credit to the original author(s) and the source, provide a link to the Creative Commons license, and indicate if changes were made.

\section{References}

1. Buchwald H, Oien DM. Metabolic/bariatric surgery worldwide 2008. Obes Surg. 2009;19(12):1605-11.

2. Sjöström L, Narbro K, Sjöström CD, et al. Effects of bariatric surgery on mortality in Swedish obese subjects. N Engl J Med. 2007;357(8):741-52.

3. Mechanick JI, Kushner RF, Sugerman HJ, et al. The Obesity Society and American Society for Metabolic \& Bariatric Surgery medical guidelines for clinical practice for the perioperative nutritional, metabolic, and nonsurgical support of the bariatric surgery patient. Obesity. 2009;17(1):1-70.
4. Gagnon LE, Karwacki Sheff EJ. Outcomes and complications after bariatric surgery: a randomized study of gastric bypass and duodenal switch. Am J Nurs. 2012;112(9):26-36.

5. Disse E, Pasquer A, Espalieu P, et al. Greater weight loss with the omega loop bypass compared to the Roux-en-Y gastric bypass: a comparative study. Obes Surg. 2014;24:841-6.

6. Lee WJ, Yu PJ, Wang W, et al. Laparoscopic Roux-en-Y versus mini-gastric bypass for the treatment of morbid obesity: a prospective randomized controlled clinical trial. Ann Surg. 2005;242:20-8.

7. Rutledge R, Walsh TR. Continued excellent results with the minigastric bypass: six-year study in 2,410 patients. Obes Surg. 2005;15:1304-8.

8. Burza MA, Romeo S, Kotronen A, et al. Long-term effect of bariatric surgery on liver enzymes in the Swedish Obese Subjects (SOS) study. PLOS One. 2013;8(3).

9. Wolf AM, Busch B, Kuhlmann HW, et al. Histological changes in the liver of morbidly obese patients: correlation with metabolic parameters. Obes Surg. 2005;15:225-37.

10. Svane MS, Madsbad S. Bariatric surgery - effects on obesity and related co-morbidities. Curr Diabetes Rev. 2014;10:208-14.

11. Hafeez S, Mohamed HA. Bariatric surgery as potential treatment for nonalcoholic fatty liver disease: a future treatment by choice or by chance? J Obes. 2013.

12. D'Albuquerque LA, Gonzalez AM, Wahle RC, et al. Liver transplantation for subacute hepatocellular failure due to massive steatohepatitis after bariatric surgery. Liver Transpl. 2008;14:881-5.

13. Sagredo $\mathrm{S}$, Brahm J, Uribe $\mathrm{M}$, et al. Acute liver failure after bariatric surgery. A case report and literature review. Gastroenterol Hepatol. 2013;36:76-80.

14. Sgambato D, Cotticelli G, de Sio I, et al. Liver failure in an obese middle-aged woman after biliointestinal bypass. World J Clin Cases. 2013;16(1):52-5.

15. Dale O, Salo M. The Helsinki Declaration, research guidelines and regulations: present and future editorial aspects. Acta Anaesthesiol Scand. 1996;40(7):771-2.

16. Fried M, Yumuk V, Oppert JM, et al. Interdisciplinary European guidelines on metabolic and bariatric surgery. Obes Surg. 2014;24(1):42-55.

17. Luger M, Kruschitz R, Langer F, et al. Effects of omega-loop gastric bypass on vitamin $\mathrm{D}$ and bone metabolism in morbidly obese bariatric patients. Obes Surg. 2015;25(6):1056-62.

18. Kruschitz R, Luger M, Kienbacher C, et al. Acute weight loss following omega-loop-gastric bypass surgery transiently impairs liver function in diabetic and non-diabetic patients. Wien Klin Wochenschr. 2014;126 Suppl 4:171-98.

19. Rutledge R. The mini-gastric bypass: experience with the first 1.274 cases. Obes Surg. 2001;11(3):276-80.

20. Pepy MB, Hirschfield GM. C-reactive protein: a critical update. J Clin Invest. 2003;111:1805-12.

21. Kotronen A, Peltonen M, Hakkarainen A, et al. Prediction of nonalcoholic fatty liver disease and liver fat using metabolic and genetic factors. Gastroenterology. 2009;137:865-72.

22. Angulo P, Hui JM, Marchesini G, et al. The NAFLD fibrosis score: a noninvasive system that identifies liver fibrosis in patients with NAFLD. Hepatology. 2007;45(4):846-54.

23. Angulo P, Keach JC, Batts KP, et al. Independent predictors of liver fibrosis in patients with nonalcoholic steatohepatitis. Hepatology. 1999;30:1356-62.

24. Wallace TM, Levy JC, Matthews DR. Use and abuse of HOMA modeling. Diabetes Care. 2004;27(6):1487-95.

25. Alberti KG, Zimmet P, Shaw J, IDF Epidemiology Task Force Consensus Group. The metabolic syndrome - a new worldwide definition. Lancet. 2005;366:1059-62.

26. Ratziu V, Giral P, Charlotte F, et al. Liver fibrosis in overweight patients. Gastroenterology. 2000;118(6):1117-23. 
27. Deitel M, Greenstein RJ. Recommandations for reporting weight loss. Obes Surg. 2003;13(2):159-60.

28. Chevallier JM, Arman GA, Guenzi M, et al. One thousand single anastomosis (omega loop) gastric bypasses to treat morbid obesity in a 7-year period: outcomes show few complications and good efficacy. obes surg. 2015;25:951-8.

29. Noun R, Skaff J, Riachi E, et al. One thousand consecutive mini-gastric bypass: short- and long-term outcome. Obes Surg. 2012;22:697-703.

30. Victorzon M. Single-anastomosis gastric bypass: better, faster, and safer? Scand J Surg. 2014;104:48-53.

31. Gasteyger C, Larsen TM, Vercruysse F, et al. Effect of a dietaryinduced weight loss on liver enzymes in obese subjects. Am J Clin Nutr. 2008;87:1141-7.

32. Promrat K, Kleiner DE, Niemeier HM, et al. Randomized controlled trial testing the effects of weight loss on nonalcoholic steatohepatitis (NASH). Hepatology. 2010;51(1):121-9.

33. Chavez-Tapia NC, Tellez-Avila FI, Barrientos-Gutierrez T, et al. Bariatric surgery for non-alcoholic steatohepatitis in obese patients. Cochrane Database Syst Rev. 2010;2010(1).

34. Luyckx FH, Desaive C, Thiry A, et al. Liver abnormalities in severely obese subjects: effect of drastic weight loss after gastroplasty. Int J Obes. 1998;22:222-6.

35. Wolf AM, Beisiegel U. The Effect of loss of excess weight on the metabolic risk factors after bariatric surgery in morbidly and superobese patients. Obes Surg. 2007;17:910-9.
36. Kim HC, Nam CM, Jee SH, et al. Normal serum aminotransferase concentration and risk of mortality from liver diseases: prospective cohort study. BMJ. 2004;328:983.

37. Clark JM, Brancati FL, Diehl AM. The prevalence and etiology of elevated aminotransferase levels in the United States. Am J Gastroenterol. 2003;98:960-7.

38. Bo S, Gambino R, Durazzo M, et al. Associations between $\gamma$ glutamyl transferase, metabolic abnormalities and inflammation in healthy subjects from a population-based cohort: a possible implication for oxidative stress. World J Gastroenterol. 2005;11(45): 7109-17.

39. Ghouri N, Preiss D, Sattar N. Liver enzymes, nonalcoholic fatty liver disease, and incident cardiovascular disease: a narrative review and clinical perspective of prospective data. Hepatology. 2010;52: 1156-61.

40. Torgerson JS, Lindroos AK, Sjöström CD, et al. Are elevated aminotransferases and decreased bilirubin additional characteristics of the metabolic syndrome? Obesity Res. 1997;5(105-114).

41. Johnston DEJ. Special considerations in interpreting liver function tests. Am Fam Physician. 1999;59(8):2223-30.

42. Fuchs CD, Claudel T, Trauner M. Role of metabolic lipases and lipolytic metabolites in the pathogenesis of NAFLD. Trends Endocrinol Metab. 2014;25(11):576-85. 